\title{
Hipotermia intravascular prolongada en un paciente con hipertensión endocraneana refractaria
}

\author{
MAXIMILIANO ROVEGNO ${ }^{1}$, JOSÉ LUIS VALENZUELA ${ }^{1}$, \\ PATRICIO MELLADO ${ }^{2}$, MAX ANDRESEN ${ }^{1}$
}

\section{Prolonged hypothermia in refractory intracranial hypertension. Report of one case}

The use of hypothermia after cardiac arrest caused by ventricular fibrillation is a standard clinical practice, however its use for neuroprotection has been extended to other conditions. We report a 23-year-old male with intracranial hypertension secondary to a parenchymal hematoma associated to acute hydrocephalus. An arterial malformation was found and embolized. Due to persistent intracranial hypertension, moderate hypothermia with a target temperature of $33^{\circ} \mathrm{C}$ was started. After 12 hours of hypothermia, intracranial pressure was controlled. After 13 days of hypothermia a definitive control of intracranial pressure was achieved. The patient was discharged 40 days after admission, remains with a mild hemiparesia and is reassuming his university studies.

(Rev Med Chile 2012; 140: 219-224).

Key words: Hematoma, subdural; Hypothermia, Induced; Intracranial Hypertension; Intracranial Arteriovenous malformations.

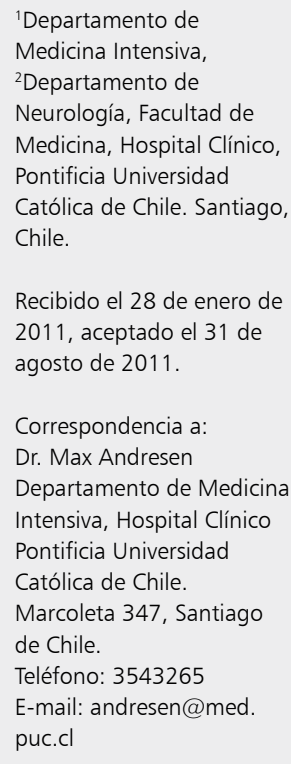

Recibido el 28 de enero de 2011, aceptado el 31 de agosto de 2011.

Correspondencia a:

Dr. Max Andresen Departamento de Medicina Intensiva, Hospital Clínico Pontificia Universidad Católica de Chile.

Marcoleta 347, Santiago de Chile.

Teléfono: 3543265

E-mail: andresen@med.

puc.cl

E l uso de hipotermia moderada como técnica de neuroprotección cuenta con una amplia evidencia básica y clínica $^{1-4}$. Su empleo se generalizó en esta última década, tras los resultados positivos de dos trabajos de distribución aleatoria que demostraron su utilidad post paro cardiorrespiratorio $(\mathrm{PCR})^{3,4}$. Hoy en día la hipotermia moderada $\left(33^{\circ} \mathrm{C}\right)$ es un estándar en los cuidados post reanimación del PCR por fibrilación ventricular o taquicardia ventricular ${ }^{5,6}$. También existe evidencia de su utilidad en la asfixia perinatal, en menor grado en el traumatismo encéfalo craneano grave y la hipertensión endocraneana (HEC) refractaria ${ }^{7}$.

El mecanismo por el cual la hipotermia protege al encéfalo es multifactorial. Incluye tanto efectos dependientes de la relación transporte-consumo de oxígeno $\left(\mathrm{O}_{2}\right)$ cerebral, como de procesos netamente tisulares ${ }^{8,9}$. Entre los primeros destaca la reducción del metabolismo cerebral, restableciendo la oferta y demanda de $\mathrm{O}_{2}$. Mientras que entre los segundos, hay disminución de la excitoxicidad, modulación de la apoptosis, disminución del edema y de la respuesta inflamatoria post lesión ${ }^{8,9}$.

El objetivo de este trabajo es comunicar nuestra experiencia con un caso clínico, donde la hipotermia prolongada, por más de 10 días, permitió esperar la resolución de la patología de base, logrando un buen resultado neurológico.

\section{Caso clínico}

Paciente hombre de 23 años sin antecedentes mórbidos que presentó cefalea ictal y compromiso de conciencia cuantitativo progresivo. Consultó inicialmente en otro hospital donde se diagnosticó mediante tomografía computarizada (TC) de encéfalo un hematoma intraparenquimatoso parietal derecho de $42 \mathrm{ml}$, asociado a hidrocefalia aguda. En pabellón y de forma urgente, se instaló un drenaje ventricular externo (DVE). Se registró 
una presión intracraneana (PIC) inicial de $45 \mathrm{~cm}$ de $\mathrm{H}_{2} 0$. Se conectó a ventilación mecánica (VM) y se indicó sedación profunda. Inicialmente, la hipertensión intracraneana se trató con drenaje de líquido cefalorraquídeo y sodio hipertónico intravenoso en bolos, según necesidad. Se trasladó a nuestro servicio tres días más tarde.

Ingresó intubado y conectado a VM, sedado con propofol $0,71 \mathrm{mg} / \mathrm{kg} / \mathrm{h}$, y hemodinamia apoyada con noradrenalina a $0,06 \mu \mathrm{g} / \mathrm{kg} / \mathrm{min}$, PIC de $6 \mathrm{~cm}$ de $\mathrm{H}_{2} \mathrm{O}$ y presión de perfusión cerebral (PPC) de $80 \mathrm{mmHg}$. En el examen neurológico se encontraba en coma, con midriasis fija a derecha, reflejos corneales abolidos, reflejos oculocefálicos esbozados, tono y reflejos osteotendíneos disminuidos, reflejos plantares indiferentes bilaterales.
Exámenes generales de ingreso: $\mathrm{Na}^{+} 146 \mathrm{mEq} / \mathrm{L}, \mathrm{K}^{+}$ $3,5 \mathrm{mEq} / \mathrm{L}$, hematocrito $31 \%$, lactato $1,4 \mathrm{mmol} / \mathrm{L}$, leucocitos 9.600 por $\mathrm{mm}^{3}$ y proteína $C$ reactiva $11,4 \mathrm{mg} / \mathrm{dl}$.

La angiografía cerebral de 6 vasos mostró una malformación arteriovenosa (MAV) parietal mesial derecha con signos de rotura reciente, asociada a tres aneurismas paranidales (Figura 1). Se realizó una embolización con N-butil-cianocrilato (Histoacryl ${ }^{\oplus}$, Braun, Alemania), logrando reducir el nido de la MAV y excluir sus aneurismas. Después del procedimiento, la TC de encéfalo mostró el Histoacryl ${ }^{\Phi}$ correctamente situado. Sin embargo, se observó un incremento del hematoma con acentuado efecto de masa y presencia de edema cerebral difuso con obliteración de los surcos de la

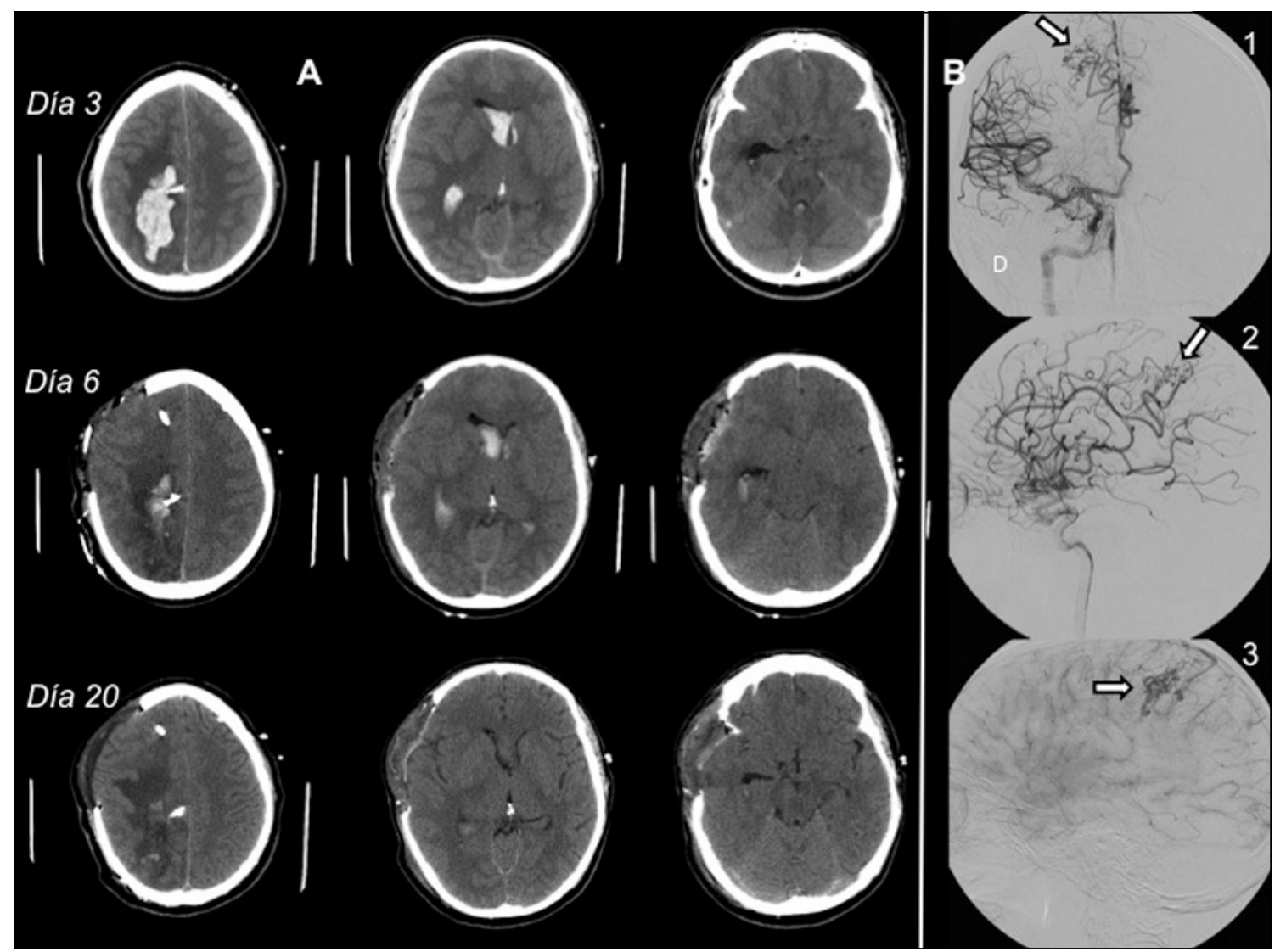

Figura 1. A: Evolución de las imágenes de TC encéfalo. En el margen derecho, se detalla el curso temporal respecto al inicio del cuadro. Día 6, corresponde al día previo al inicio de la hipotermia y en el día 20, se logra suspenderla. B: Angiografía cerebral por sustracción digital. 1: Proyección anterior, fase arterial, " $D$ " indica derecha. 2 y 3: Proyección lateral derecha, fases arteria y venosa respectivamente. Fechas blancas muestran una MAV parietal derecha con un nido de $\sim 13 \mathrm{~mm}$, dependiente de ramas posteriores de la arteria cerebral anterior derecha. El drenaje venoso se realiza al seno longitudinal superior. 
convexidad, además de herniaciones subfalcina y uncal con desviación de la línea media de $10 \mathrm{~mm}$, a nivel de la glándula pineal. Se realizó la evacuación del hematoma y una craniectomía descompresiva frontoparietoccipital derecha extensa de 10,2 x 9,3 $\mathrm{cm}$. La TC de encéfalo de control al día siguiente mostró una reducción del hematoma a $11 \mathrm{ml}$, pero aún se mantenía un importante efecto de masa, con desviación de la línea media de $5 \mathrm{~mm}$.

A pesar de la craniectomía evolucionó con PIC mayores a $20 \mathrm{~cm} \mathrm{H}_{2} \mathrm{O}\left(23 \pm 5 \mathrm{~cm} \mathrm{H}_{2} \mathrm{O}\right.$; media \pm DS). Se instauró un manejo médico escalonado de la HEC, con sedación profunda (fentanilo 2,86 $\mu \mathrm{g} / \mathrm{kg} / \mathrm{h}$, midazolam $0,11 \mathrm{mg} / \mathrm{kg} / \mathrm{h}$ y propofol 1,43 $\mathrm{mg} / \mathrm{kg} / \mathrm{h}$ ), bloqueo neuromuscular (vecuronio $0,09 \mathrm{mg} / \mathrm{kg} / \mathrm{h}$ ), además del uso frecuente de bolos de sodio hipertónico frente a cifras de PIC > $30 \mathrm{~cm} \mathrm{H_{2 }} \mathrm{O}$. Se descartó actividad epiléptica no convulsiva mediante un electroencefalograma y la TC de encéfalo 48 h post craniectomía no mostró cambios significativos. Debido a la persistencia

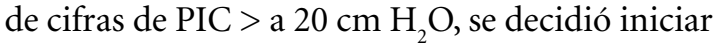
hipotermia terapéutica.

Se empleó hipotermia moderada, con temperatura objetivo de $33^{\circ} \mathrm{C}$, mediante técnica intravascular (Alsius, Zoll, Chelmsford, Massachusetts, EEUU). Se midió la temperatura central con catéter intravesical. Una vez conseguida la hipotermia $(\sim 12 \mathrm{~h}$ desde el diagnóstico de HEC refractaria), se logró un rápido control de la $\mathrm{PIC}$ con cifras entre 9 a $12 \mathrm{~cm} \mathrm{H}_{2} \mathrm{O}(\sim 3$ desde el inicio del procedimiento). El bloqueo neuromuscular se mantuvo en infusión continua los primeros dos días de hipotermia, para evitar los calofríos.

Luego de $48 \mathrm{~h}$ en hipotermia, se realizaron intentos programados de recalentamiento a una tasa de $0,5^{\circ} \mathrm{C} / \mathrm{h}$. Sin embargo, todos ellos fueron interrumpidos por rebote de la PIC (Figura 2). A partir del día 20 de evolución del cuadro clínico y coincidiendo con una disminución del edema cerebral en las neuroimágenes (Figura 1), se logró el control definitivo de la PIC. Completó $\sim 13$ días en hipotermia moderada, sin evidenciarse arritmias, trastornos de la coagulación o electrolíticos. Presentó una traqueobronquitis asociada a ventilación mecánica, resuelta satisfactoriamente con antibioticoterapia.

Se suspendió progresivamente la sedación, el paciente adquirió vigilia y se objetivó una hemiparesia leve con heminegligencia y hemianopsia homónima izquierda. Una vez extubado se constató lenguaje apropiado. Se inició neurorrehabilitación y finalmente, luego de 40 días de hospitalización, fue dado de alta. Dos años después se encuentra

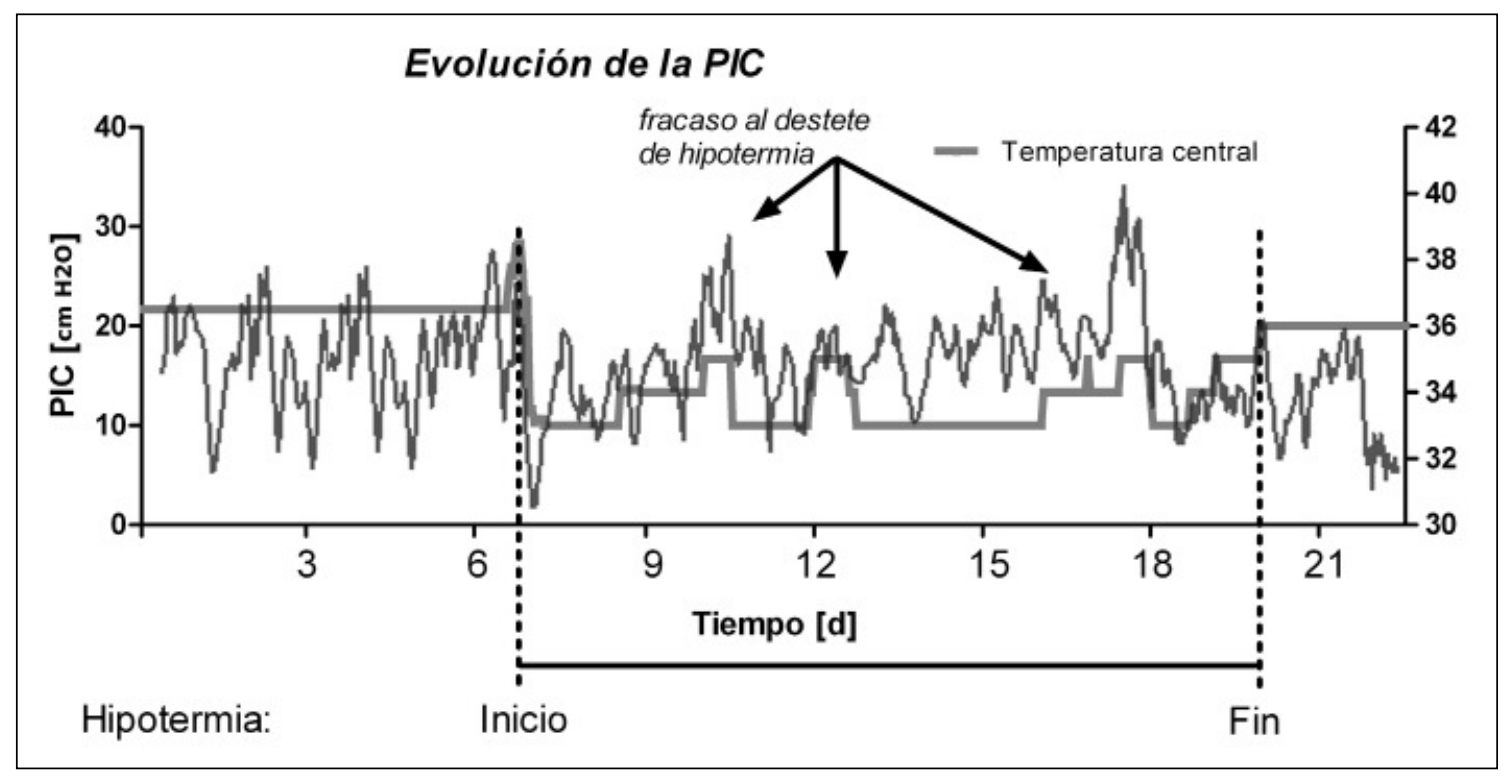

Figura 2. Correlación temporal de la PIC versus la temperatura central. La escala tiempo esta referida al inicio del cuadro. Se observa como la reducción de la temperatura a $33^{\circ} \mathrm{C}$, logra normalizar las cifras de PIC y quedan graficados los rebotes de HEC, durante los intentos de recalentamiento. 
en Rankin 2, con hemiparesia izquierda espástica leve y completando sus estudios universitarios.

\section{Discusión}

El presente caso corresponde a una hemorragia intracraneana por una MAV, que representa el $1,4 \%$ de los accidentes vasculares ${ }^{10}$. Afecta principalmente a la población adulta joven, como en nuestro paciente ${ }^{11}$.

La irrupción de sangre en el parénquima cerebral genera un hematoma, con efecto de masa, disminuyendo la distensibilidad cerebral. Paralelamente, se activan mecanismos inflamatorios y de apoptosis-necrosis, que culminan con el desarrollo de un edema cerebral, lo cual deteriora aun más la distensibilidad cerebral, elevando finalmente la PIC.

Nuestro paciente presentó ese perfil fisiopatológico. De hecho, en el examen neurológico de ingreso se encontraba en coma y con midriasis fija a derecha. En ausencia de medicamentos midriáticos y ciclopléjicos locales, este hallazgo era concordante con la herniación uncal que existía. Dado el elevado efecto de masa que ejerció el hematoma, la evacuación del hematoma era indispensable. La craniectomía descompresiva fue complementaria a la evacuación, con la intención de resolver la HEC. Sin embargo, fue insuficiente, como lo demostró la evolución de la PIC post neurocirugía. El manejo escalonado de la HEC que empleamos es el actualmente recomendado en la literatu$\mathrm{ra}^{12,13}$ : las medidas de primer orden comprenden el uso de sedación, como la que utilizamos y en un segundo escalón se incluye el uso de bloqueo neuromuscular y osmoterapia. La sedación y el bloqueo neuromuscular disminuyen la PIC al reducir las demandas metabólicas, la asincronía paciente-ventilador, la congestión venosa central, y las respuestas adrenérgicas ${ }^{14}$. La osmoterapia con soluciones salinas hipertónicas disminuye el edema cerebral al aumentar la tonicidad del volumen sanguíneo cerebral, y favorecer el drenaje del agua desde el espacio extravascular ${ }^{15}$. Pese al uso de estas medidas, se evidenció que el manejo médico era ineficaz para normalizar la PIC.

Es en este escenario de HEC refractaria donde iniciamos la hipotermia moderada. Claramente, ésta tuvo un profundo impacto en controlar las cifras de PIC (Figura 2).

La hipotermia disminuye el metabolismo ce- rebral y el edema cerebral citotóxico. Se produce una disminución del volumen sanguíneo cerebral, que no compromete el aporte de nutrientes, dada la disminución de los requerimientos. Todo lo cual determina una disminución de la PIC ${ }^{8,9}$.

Smrcka M. y cols., en un estudio de 72 pacientes con TEC grave (Glasgow $<8$ ), demostró que el empleo de hipotermia moderada logró disminuir la PIC, tanto en las lesiones cerebrales primarias como en los casos con hematomas extracerebrales $^{16}$. Una reciente revisión Cochrane analizó 21 trabajos, que incluyeron 1.587 pacientes con TEC aleatorizados a hipotermia moderada o tratamiento convencional ${ }^{17}$. Los pacientes tratados con hipotermia tuvieron menos resultados desfavorables (muerte, estado vegetativo o discapacidad severa), que los del grupo control (OR 0,69; IC 95\% 0,55-0,86 $)^{17}$.

En relación a su eficacia, Schreckinger, M. y cols., en una revisión de la literatura, compararon el empleo de hipotermia con otras medidas convencionales para la HEC post $\mathrm{TEC}^{18}$. En un total de 16 estudios, que incluyeron un grupo control con normotermia, se analizaron 367 pacientes. La hipotermia logró reducir la PIC en 9,97 \pm 6,66 $\mathrm{mmHg}$, lo que fue tan efectivo como otras terapias convencionales: hiperventilación, manitol o barbitúricos ${ }^{18}$.

El uso de hipotermia no está exento de complicaciones. Nielsen $\mathrm{N}$ y cols analizaron la incidencia de complicaciones en el registro americano-europeo de hipotermia post PCR. En 765 pacientes tratados entre el 2004 y 2008 , se encontró $48 \%$ de neumonías, $37 \%$ de alteraciones hidroelectrolíticas, $14 \%$ de arritmias, y $6 \%$ de hemorragias asociadas al empleo de hipotermia ${ }^{19}$.

No todas las complicaciones asociadas a la hipotermia tienen una relación causa efecto con ella. De hecho, su uso requiere el empleo de una sedación profunda y muchas veces asociada a bloqueo neuromuscular, para evitar los calofríos que aumentan significativamente el consumo de oxígeno $^{7,20}$. Sólo la sedación y el bloqueo neuromuscular, administrados en forma protocolizada contra un objetivo de sedación moderada, han demostrado reducir el número de días en ventilación mecánica ${ }^{21}$ y la probabilidad de neumonía asociada al ventilador mecánico $(\mathrm{NAV})^{22}$. Sin embargo, su uso durante hipotermia está asociado a un objetivo de sedación profunda ${ }^{23}$, por lo que puede contribuir tanto al aumento de $\mathrm{NAV}^{24}$ 
como de polineuromiopatía ${ }^{25}$. En nuestro paciente, limitamos el uso de vecuronio a los episodios de calofríos que ocurrieron principalmente durante los primeros días de la hipotermia. Es posible que esta estrategia haya evitado el desarrollo de NAV o polineuromiopatía.

Nuestro caso refleja la eficacia de la hipotermia descrita para el manejo de la $\mathrm{HEC}^{18}$. Nuestra intención fue prolongar su uso por $48 \mathrm{~h}$, pero estuvimos obligados a mantenerla por 13 días, debido a los rebotes de HEC durante los intentos de recalentamiento. Visto en retrospectiva, sólo la disminución del edema cerebral marcó un cambio en la evolución. Lo cual hace sentido con la resolución de la patología, y una mejoría de la distensibilidad cerebral. Nuestro paciente no experimentó complicaciones significativas, logrando una buena recuperación neurológica, a pesar de lo prolongado de la hipotermia.

En los cuidados post PCR, está demostrado que se requieren al menos $12 \mathrm{~h}$ de hipotermia ${ }^{3,4}$, mientras que el uso de hipotermia por más de $48 \mathrm{~h}$ en pacientes con HEC refractaria sólo ha sido estudiado en el $\mathrm{TEC}^{26} \mathrm{y}$ en la falla hepática fulminante ${ }^{27}$. Sin embargo, dado que la acción neuroprotectora de la hipotermia es multifactorial, es muy probable que se obtenga beneficio en enfermedades con una fisiopatología similar, donde los mecanismos de daño ejercen su efecto por más de $48 \mathrm{~h}^{28}$. La duración de la hipotermia en el TEC, es un punto debatido en la actualidad. Un trabajo aleatorizado exploró el uso de hipotermia moderada por 2 días versus 5 días. Se estudiaron 215 pacientes con TEC grave y HEC. El 43,5\% de los pacientes en hipotermia prolongada $(5 \pm 1,3$ días) tuvo un resultado neurológico favorable, comparado con el $29 \%$ de los pacientes en hipotermia no prolongada $(2 \pm 0,6$ días $) \mathrm{p}<0,05^{29}$. Entre las ventajas de la hipotermia por más de $48 \mathrm{~h}$, se encontró una menor tendencia al rebote de las cifras de PIC, durante el recalentamiento ${ }^{29}$. Peterson y cols, en un meta análisis que incluyó ocho trabajos, observó que el uso de hipotermia redujo la mortalidad en $20 \%$, pero sin alcanzar significación estadística (RR 0,80; 95\% IC 0,59$1,09)^{30}$. Sin embargo, en un análisis de subgrupos, demostró que existía una diferencia significativa a favor de la hipotermia cuando ésta se empleaba por más de 48 h (RR 0,51; 95\% IC 0,33-0,79) ${ }^{30}$. Actualmente, esta en curso el "Eurotherm", un trabajo aleatorizado y multicéntrico de hipotermia por más de $48 \mathrm{~h}$, que probablemente defina el beneficio de la hipotermia prolongada en el $\mathrm{TEC}^{31}$.

\section{Referencias}

1. Lotocki G, Rivero Vaccari JP de, Pérez ER, Alonso OF, Curbelo K, Keane RW, et al. Therapeutic hypothermia modulates TNFR1 signaling in the traumatized brain via early transient activation of the JNK pathway and suppression of XIAP cleavage. Eur J Neurosci 2006; 24: 2283-90.

2. Meybohm P, Gruenewald M, Zacharowski KD, Albrecht M, Lucius R, Fösel N, et al. Mild hypothermia alone or in combination with anesthetic post-conditioning reduces expression of inflammatory cytokines in the cerebral cortex of pigs after cardiopulmonary resuscitation. Crit Care 2010; 14: R21.

3. Bernard SA, Gray TW, Buist MD, Jones BM, Silvester W, Gutteridge G, et al. Treatment of comatose survivors of out-of-hospital cardiac arrest with induced hypothermia. N Engl J Med 2002; 346: 557-63.

4. Hypothermia after Cardiac Arrest Study Group. Mild therapeutic hypothermia to improve the neurologic outcome after cardiac arrest. N Engl J Med 2002; 346: 549-56.

5. Nolan J. European Resuscitation Council guidelines for resuscitation 2005. Section 1. Introduction. Resuscitation 2005; 67: S3-6.

6. 2005 American Heart Association Guidelines for Cardiopulmonary Resuscitation and Emergency Cardiovascular Care. Circulation 2005; 112: IV1-203.

7. Varon J, Acosta P. Therapeutic hypothermia: past, present, and future. Chest 2008; 133: 1267-74.

8. Holzer M. Targeted temperature management for comatose survivors of cardiac arrest. N Engl J Med 2010; 363: 1256-64.

9. Polderman KH. Mechanisms of action, physiological effects, and complications of hypothermia. Crit Care Med 2009; 37: S186-202.

10. Stapf C, Labovitz DL, Sciacca RR, Mast H, Mohr JP, Sacco RL. Incidence of adult brain arteriovenous malformation hemorrhage in a prospective population-based stroke survey. Cerebrovasc Dis 2002; 13: 43-6.

11. ApSimon HT, Reef H, Phadke RV, Popovic EA. A population-based study of brain arteriovenous malformation: long-term treatment outcomes. Stroke 2002; 33: 2794800.

12. Carney NA, Ghajar J. Guidelines for the management of severe traumatic brain injury. Introduction. J Neurotrauma 2007; 24: S1-2.

13. Latorre JGS, Greer DM. Management of acute intra- 
cranial hypertension: a review. Neurologist 2009; 15: 193-207.

14. Lassen NA, Christensen MS. Physiology of cerebral blood flow. Br J Anaesth 1976; 48: 719-34.

15. Wolfe TJ, Torbey MT. Management of intracranial pressure. Curr Neurol Neurosci Rep 2009; 9: 477-85.

16. Smrcka M, Vidlák M, Máca K, Smrcka V, Gál R. The influence of mild hypothermia on ICP, CPP and outcome in patients with primary and secondary brain injury. Acta Neurochir Suppl 2005; 95: 273-5.

17. Sydenham E, Roberts I, Alderson P. Hypothermia for traumatic head injury Cochrane Database of Systematic Reviews 2009; 2: CD001048.

18. Schreckinger M, Marion DW. Contemporary Management of Traumatic Intracranial Hypertension: Is There a Role for Therapeutic Hypothermia? Neurocritical care. 2009; 11: 427-36.

19. Nielsen N, Sunde K, Hovdenes J, Riker RR, Rubertsson $\mathrm{S}$, Stammet $\mathrm{P}$, et al. Adverse events and their relation to mortality in out-of-hospital cardiac arrest patients treated with therapeutic hypothermia. Crit Care Med 2010; 39: 57-64.

20. Horvath SM, Spurr GB, Hutt BK, Hamilton LH. Metabolic cost of shivering. J Appl Physiol 1956; 8: 595-602.

21. Girard TD, Kress JP, Fuchs BD, Thomason JWW, Schweickert WD, Pun BT, et al. Efficacy and safety of a paired sedation and ventilator weaning protocol for mechanically ventilated patients in intensive care (Awakening and Breathing Controlled trial): a randomised controlled trial. Lancet 2008; 371: 126-34.

22. Quenot J-P, Ladoire S, Devoucoux F, Doise J-M, Cailliod $\mathrm{R}$, Cunin N, et al. Effect of a nurse-implemented sedation protocol on the incidence of ventilator-associated pneumonia. Crit Care Med 2007; 35: 2031-6.
23. Seder DB, Van der Kloot TE. Methods of cooling: practical aspects of therapeutic temperature management. Crit Care Med 2009; 37: S211-22.

24. Cook DJ, Walter SD, Cook RJ, Griffith LE, Guyatt GH, Leasa $\mathrm{D}$, et al. Incidence of and risk factors for ventilator-associated pneumonia in critically ill patients. Ann Intern Med 1998; 129: 433-40.

25. Jonghe B de, Lacherade J-C, Sharshar T, Outin H. Intensive care unit-acquired weakness: risk factors and prevention. Crit Care Med 2009; 37: S309-15.

26. Jiang J-Y. Clinical study of mild hypothermia treatment for severe traumatic brain injury. J Neurotrauma 2009; 26: 399-406.

27. Jacob S, Khan A, Jacobs ER, Kandiah P, Nanchal R. Prolonged hypothermia as a bridge to recovery for cerebral edema and intracranial hypertension associated with fulminant hepatic failure. Neurocrit Care 2009; 11: 2426.

28. Werner C, Engelhard K. Pathophysiology of traumatic brain injury. Br J Anaesth 2007; 99: 4-9.

29. Jiang J-Y, Xu W, Li W-P, Gao G-Y, Bao Y-H, Liang Y-M, et al. Effect of long-term mild hypothermia or shortterm mild hypothermia on outccome of patients with severe traumatic brain injury. J Cerb Blood Flow Metab 2006; 26:771-6.

30. Peterson K, Carson S, Carney N. Hypothermia treatment for traumatic brain injury: a systematic review and meta-analysis. J Neurotrauma 2008; 25: 62-71.

31. Andrews PJD, Sinclair HL, Battison CG, Polderman KH, Citerio G, Mascia L, et al. European society of intensive care medicine study of therapeutic hypothermia $\left(32-35^{\circ}\right.$ C) for intracranial pressure reduction after traumatic brain injury (the Eurotherm3235Trial). Trials 2011; 12: 8 . 